Perspective

\title{
Hidden but Possibly Fatal Misconceptions in Photocatalysis Studies: A Short Critical Review
}

\author{
Bunsho Ohtani \\ Institute for Catalysis, Hokkaido University, Sapporo 001-0021, Japan; ohtani@cat.hokudai.ac.jp; \\ Tel.: +81-11-706-9132; Fax: +81-11-706-9133 \\ Academic Editor: Keith Hohn \\ Received: 31 October 2016; Accepted: 30 November 2016; Published: 2 December 2016
}

\begin{abstract}
This short review paper shows some misconceptions hidden in the discussion on the mechanism of heterogeneous photocatalysis, which may lead to fatal errors in conclusions. Topics described in this review are semiconductor photocatalysis, control experiments for proof of photocatalysis, and converse-proposition pitfalls in discussion.
\end{abstract}

Keywords: semiconductor photocatalysis; control experiment; killer card; converse proposition; logics

\section{Introduction}

As described in a critical review in this journal previously [1], the present author remains frustrated that the intrinsic mechanism of heterogeneous photocatalysis has not yet been clarified, despite the fact that the author has worked mainly in the field of heterogeneous photocatalysis for more than 30 years since starting research work for a Ph.D. degree in 1981 and has published more than 200 original and review papers on photocatalysis! Thus, what is the source of the author's frustration? Possible reasons, mainly misunderstandings, misconceptions, and/or speculation in a scientific sense, have been pointed out in the present author's recent review papers [2-5]. This short review presents new misconceptions often found in discussions of photocatalysis studies that have not yet been recognized by such papers' authors.

\section{2. "Semiconductor" Photocatalysis}

Heterogeneous photocatalysis in which a particulate photocatalyst is suspended in a solution or placed in contact with a substrate (substrates) in a gas phase and irradiated has been one of the important topics in chemical, energy-related, and environmental sciences. This heterogeneous photocatalysis has often been called "semiconductor photocatalysis". This might be because most known/used photocatalysts, e.g., titanium(IV) oxide (titania) and cadmium sulfide, are categorized as semiconductors among materials. In the history of photocatalysis studies, the discovery of photoassisted electrochemical splitting of water with a single-crystal titania electrode and a platinum counter electrode under electrochemical/chemical bias potential application [6] has accelerated research on heterogeneous photocatalysis using particulate metal oxides. In electrochemical cells, semiconductor single-crystal electrodes reduced to yield conductivity by introducing donor levels between the conduction band (CB) and the valence band have been used. When these electrodes of n-type semiconductors are in contact with electrolyte solutions, electrons in the donor levels flow out, due to their relatively high Fermi level, to the solution to produce "a depletion layer" of an electric field, where a photoexcited electron in the $\mathrm{CB}$ and a positive hole in the VB migrate in opposite directions: electrons to the surface and holes to the bulk (Figure 1). 

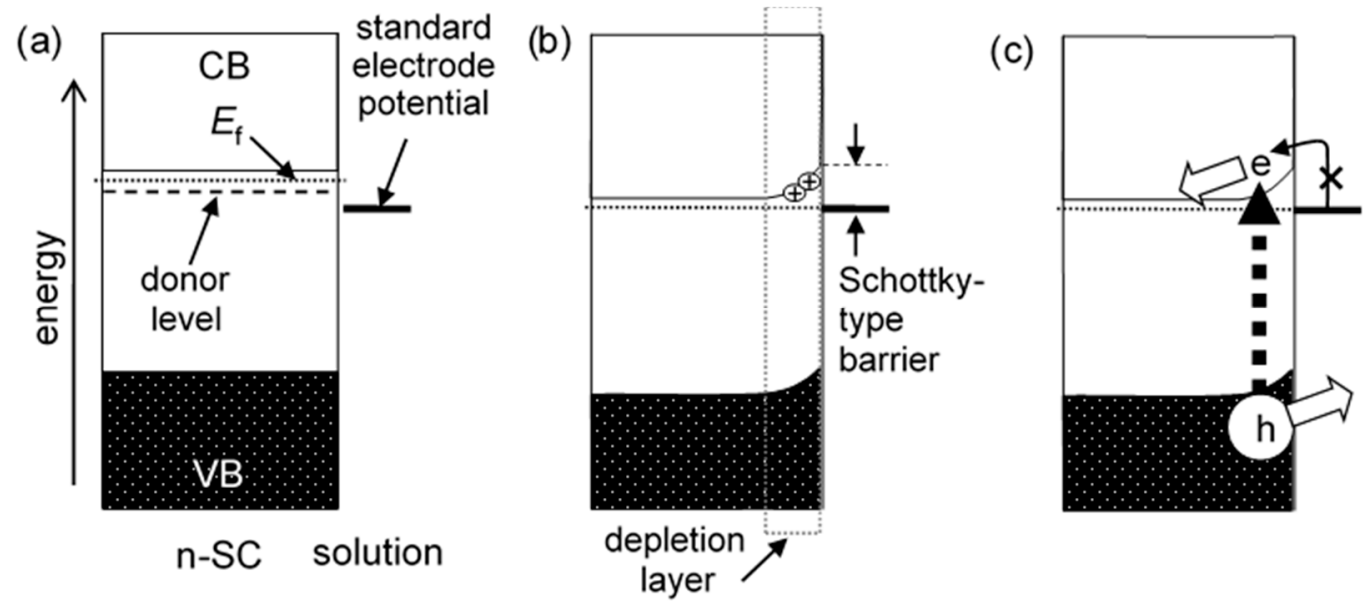

Figure 1. (a) N-type semiconductor (n-SC) solution. (b) Schottky-type junction with a depletion layer in which electrons in donor levels flow out into the solution to lower the Fermi level $\left(E_{\mathrm{F}}\right)$ and (c) n-SC-electrolyte interface under photoirradiation.

This Schottky-type barrier is one of the most significant features of a semiconductor-electrolyte solution interface. It was believed, at least in the 1980s, that the depletion layer is the reason for the high efficiency of "semiconductor photocatalysis". In this sense, it seemed reasonable to call heterogeneous photocatalysis "semiconductor photocatalysis." However, it was pointed out in the 1990s that the density of impurities for donor levels in ordinary particulate photocatalysts is too low to establish an electric field gradient within the particles, i.e., the expected depth of the depletion layer is larger than the actual particle size. Thus, Schottky-type barrier formation in the photocatalyst-solution interface has not been assumed, and a simple mechanism, as shown in Figure 2, in which reduction and oxidation by photoexcited electrons and positive holes, respectively, proceed with surface-adsorbed substrates has been used for the interpretation of heterogeneous photocatalysis in almost all of the recent publications in this field. It should be noted that characteristics of (n-type) semiconductors disappeared in this simple model, i.e., the photocatalyst materials can be insulators having an electronic structure, similar to semiconductors, composed of a filled VB and vacant CB. Thus, how can we explain the very low or negligible photocatalytic activities of insulators such as aluminum oxide (alumina) and silicon oxide (silica)?

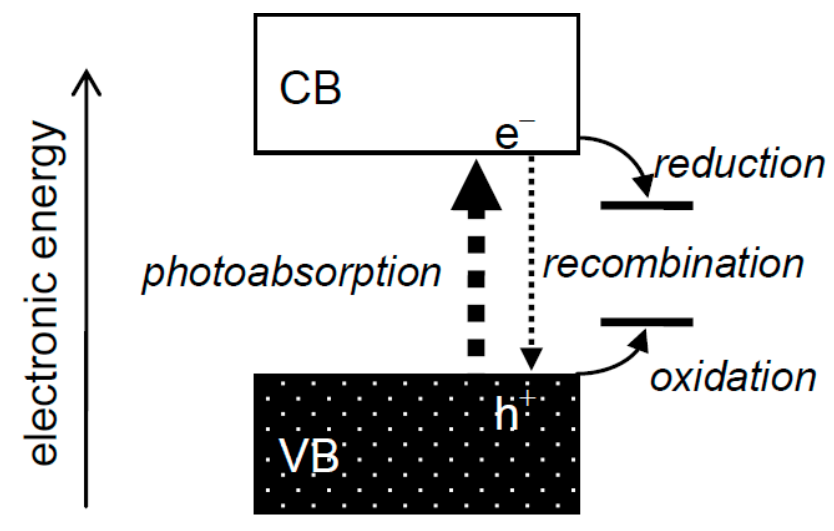

Figure 2. Commonly used interpretation of heterogeneous photocatalysis in recent years [1]. $C B$, conduction band; VB, valence band.

As has been proposed in the author's review in this very journal [1], it can be assumed that semiconducting properties, especially n-type ones, that produce electron traps (ETs) are essential for 
particulate photocatalysts. Although the density in the particulate photocatalysts described above might be very low, impurities (or surface structure) to provide donor levels may exist at least at a detectable level, and electrons in those levels may flow out, similar to those in semiconductor electrodes, to an electrolyte solution that is in contact with photocatalyst particles to result in the formation of electron-deficient vacant levels, i.e., ETs to accept photoexcited electrons. If the depth, i.e., the difference in energy between the CB bottom (CBB) and ETs, is smaller than the thermal energy of electrons $(0.026 \mathrm{eV}=26 \mathrm{meV}$ at $300 \mathrm{~K})$, electrons in these shallow traps can be thermally excited to the $\mathrm{CB}$ and be trapped again in another ET, i.e., electrons can migrate through the $\mathrm{CB}$ and shallow ETs at a speed that might be faster than electron hopping between ETs [7]. On the other hand, if ETs are located deeply, electrons once trapped by these deep ETs cannot be excited thermally to the $\mathrm{CB}$ and simply recombine with a positive hole. Thus, as a working hypothesis, it can be assumed that shallow and deep ETs enhance and reduce photocatalytic activity of particles by migration of electrons through CB-ETs and electron-positive hole recombination, respectively. In this sense, photocatalysts should be of semiconductors but not insulators possessing no ETs, and the energy-resolved distribution of ETs (ERDT) in particulate photocatalysts may be one of the decisive factors governing their photocatalytic activities.

It has been reported in a recent paper from the present author's group that ERDT as well as the $\mathrm{CBB}$ as a function of energy from the VB top can be measured by reversed double-beam photocatalytic spectroscopy (RDB-PAS), in which electrons in the VB are directly excited to ETs, and the accumulation of electrons filling ETs from the deep-energy side to shallow-energy side is recorded [8]. The ERDT/CBB patterns of more than 30 commercially or non-profitably available titania samples showed that all of the titania samples possess shallow ETs near the CBB and detectable/negligible deep ETs, and those ERDT/CBB patterns were different. Furthermore, several samples of metal oxides other than titania, e.g., tungsten(VI) oxide and cerium(IV) oxide, showed ERDT/CBB patterns, suggesting that metal oxides used as photocatalysts have ETs, presumably reflecting their n-type semiconductor properties.

\section{Control Experiments as Proof of Photocatalysis}

When the present author started working in the field of photocatalysis in 1981, it seemed to have already been believed by the scientists in the field that the photocatalysis, in the sense of the above-mentioned mechanism, can be concluded by showing the results of following "control experiments", i.e., the reaction proceeds only when a solid material, a photocatalyst, in contact with a substrate is photoirradiated, i.e., no reaction is observed in the dark even in the presence of the solid material and substrate or in the absence of the solid material or substrate even under photoirradiation. After more than 30 years, it seems that this type of control experiment is still alive; the conclusion of "photocatalysis through excitation of a solid material (a photocatalyst)" using the results of this control experiment has been presented in many recent papers.

During the author's study on action-spectrum analysis of photocatalytic reactions [9-11], it was found that the action spectrum, in the visible-light region, of decomposition (fading) of methylene blue (MB) with bare and sulfur-doped titania samples resembles the photoabsorption spectrum of $\mathrm{MB}$ adsorbed on titania surfaces [12]. This clearly indicates that decomposition of MB proceeds at least under visible-light irradiation through photoabsorption of $\mathrm{MB}$ but not of titania particles. It is plausible that the photoexcited MB adsorbed on the surface injects electrons to titania (with ETs) and that the resultant electron-deficient $\mathrm{MB}$ is self-decomposed and the injected electrons in titania reduces oxygen molecules on the titania surface. A significant point is that this "dye-sensitized mechanism" [13] requires a solid material for electron injection; therefore, such a reaction does not proceed in the absence of a solid material accepting electrons. In other words, the dye-sensitized reaction systems may satisfy the above-mentioned control experiment; negligible decomposition (fading) is observed in the absence of a solid material, an expected "photocatalyst", i.e., the control experiment cannot exclude 
such a "non-photocatalytic" mechanism. A paper from the present author's laboratory claimed that action-spectrum analysis is the only possible method of excluding a non-photocatalytic mechanism.

Thus, do we need to consider the exception(s) in the above-mentioned control experiment? Are there any other exceptions? The author found the answer for these questions very recently, i.e., after more than 30 years after the author learned of the control experiment. The answer is simple. There are possible exceptions to the control experiment, but we need not look for those exceptions because the proposition in the control experiment, that a reaction proceeding only under photoirradiation of a solid material with a substrate is a photocatalytic reaction through photoexcitation of the solid material, is logically "false." The logic is shown in Figure 3. In logic, a proposition is "if (in the case of) A, then B $(\mathrm{A} \rightarrow \mathrm{B})$. " The proposition that, if the reaction proceeds through photoexcitation of a solid material, photoirradiation, the solid material, and a substrate are all required (top right) is correct, i.e., this proposition is "true", since this is the definition of "photocatalysis". However, the converse proposition (top left), if photoirradiation, a solid material and a substrate are all required, the reaction proceeds through photoexcitation of the solid material, i.e., the logic of the control experiment, is uncertain. Thus, we cannot discuss the mechanism based on the control experiment in a logical sense!

Judgement of whether a given proposition is true or false is not straightforward, but it is well understood that a proposition is judged to be false when at least one inconsistent fact is found. For the proposition for the control experiment (top left), a contradiction "killer card" that shows the inconsistency of the proposition is the fact that a non-photocatalytic reaction proceeds only under the photoirradiation of a solid material with a substrate, and the above-mentioned action spectrum-analysis study has revealed the presence of the killer card to conclude that the control-experiment proposition is false. A question then arises. Did we need to wait for the appearance of this killer card? Logically speaking, the answer is, Of course. We need not wait but must discard the proposition, i.e., the control experiment, since the proposition of the control experiment must be uncertain without seeing the killer card. In other words, we should recognize the control experiment as one of the requirements for "photocatalysis".

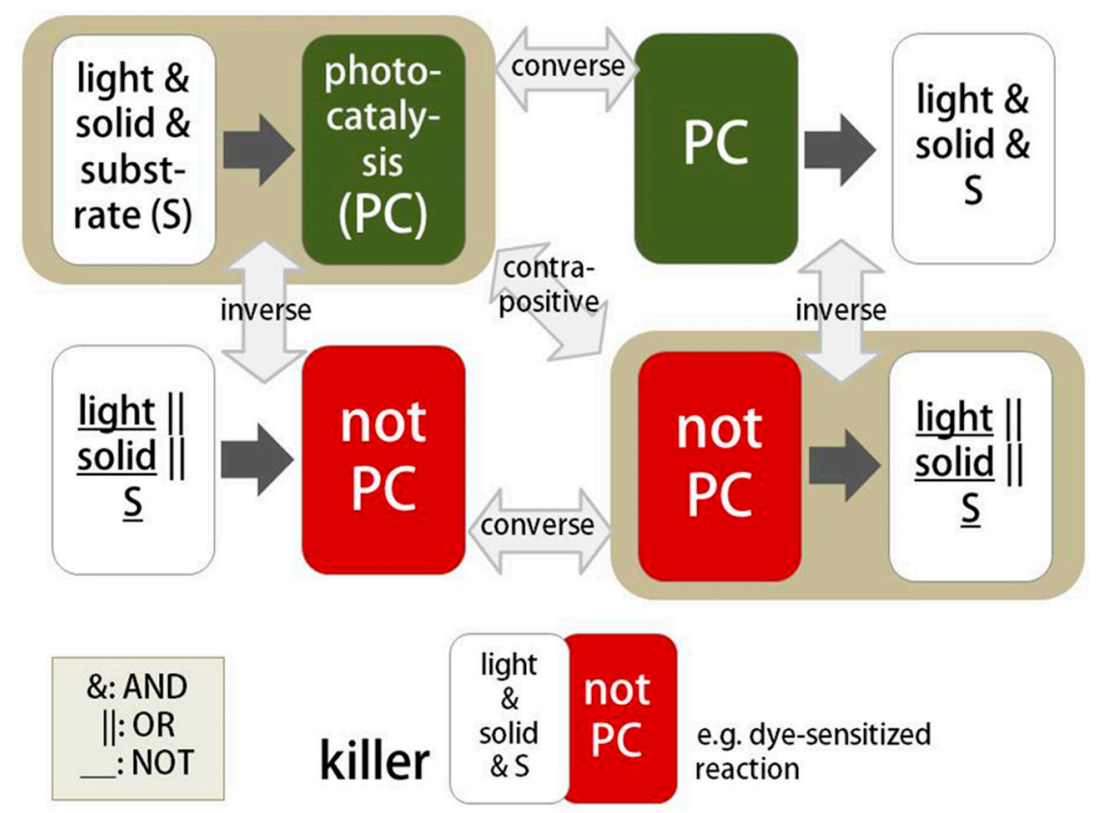

Figure 3. Propositions relating to the control experiment for proof of semiconductor photocatalysis.

\section{Converse-Proposition Pitfalls}

As discussed in the preceding section, converse and inverse propositions are uncertain even when the original proposition is true; i.e., $\mathrm{B} \rightarrow \mathrm{A}$ cannot be true even if $\mathrm{A} \rightarrow \mathrm{B}$ is true. This logic might be 
well understood by scientists, not just those working in the field of photocatalysis. The following are examples for checking this converse-proposition logic (or "affirming-the-consequence fallacy" [14]).

It is reasonable to expect that when one photocatalyst particle absorbs a multiple number of photons to store four positive holes to be used in four-electron transfer to two water molecules, oxygen, but not hydrogen peroxide, is liberated.

A1: four-electron transfer in one photocatalyst particle

B1: oxygen, but not hydrogen peroxide, liberation

Many papers on photocatalysis have claimed that, if a photocatalyst has a smaller number of defective sites that induce electron positive-hole recombination, higher quantum efficiency is observed.

A2: fewer defective sites that induce electron-hole recombination

B2: higher quantum efficiency

It is probable that, if a photocatalyst has higher specific surface area for high-density substrate adsorption, higher photocatalytic activity can be expected due to the resultant higher surface density of reaction substrates [15].

A3: high specific surface area for high-density substrate adsorption

B3: higher photocatalytic activity

It has often been claimed, especially for anatase rutile-mixed Evonik (Degussa P25), that, if electron (hole) transfer between anatase and rutile particles occurs, higher photocatalytic activity of the anatase-rutile mixture is obtained.

A4: electron (hole) transfer between anatase and rutile particles

B4: higher photocatalytic activity of anatase-rutile mixture

In all of the cases, propositions $\mathrm{A} \rightarrow \mathrm{B}$ may be true, since they are of definition or expectation. One problem is that converse propositions $B \rightarrow A$ must be uncertain, even if the original propositions $\mathrm{A} \rightarrow \mathrm{B}$ are true: (1) oxygen can be liberated as a sole oxidation product via stepwise reaction including hydrogen peroxide as an intermediate; (2) higher quantum efficiency can be attributed to faster migration of electrons or positive holes [16,17]; (3) higher photocatalytic activity is attributable to fewer defective sites [16,17]; and (4) each of the crystallites of anatase and rutile show higher photocatalytic activity even without electron (hole) transfer between anatase and rutile particles [18]. In other words, other propositions $\mathrm{C} \rightarrow \mathrm{B}, \mathrm{D} \rightarrow \mathrm{B}$ or others, may be true.

\section{Conclusive Remarks}

Again, despite extensive studies having been carried out for at least 40 years since the first-generation boom following the publication of a paper by Fujishima and Honda [6], we have not yet unraveled the fundamentals of photocatalysis, as pointed out in the author's review [1]. One of the possible reasons pointed out in this review is that we have studied shared understandings and/or beliefs for so long and have assumed them to be common sense without checking them logically and scientifically. What surprised the author-indeed, one of the motivations of writing this review-was the converse-proposition pitfall for the control experiment; the author believed that he and collaborators had found the exception: non-photocatalytic reactions passing the control experiments. However, the presence of such a killer card had already been predicted. Although we scientists may believe that we can think and discuss logically (without any proof and guarantees), we need to be logical "intentionally", since human beings cannot be mechanically/truly logical as “Wason's four-card problem" suggests [19].

Conflicts of Interest: The author declares no conflict of interest. 


\section{References}

1. Ohtani, B. Titania photocatalysis beyond recombination: A critical review. Catalysts 2013, 3, $942-953$. [CrossRef]

2. Ohtani, B. Preparing articles on photocatalysis-Beyond the illusions, misconceptions and speculation. Chem. Lett. 2008, 37, 216-229. [CrossRef]

3. Ohtani, B. Photocatalysis A to Z-What we know and what we don't know. J. Photochem. Photobiol. C 2010, 11, 157-178. [CrossRef]

4. Ohtani, B. Revisiting the fundamental physical chemistry in heterogeneous photocatalysis: Its thermodynamics and kinetics. Phys. Chem. Chem. Phys. 2014, 16, 1788-1797. [CrossRef] [PubMed]

5. Ohtani, B. Revisiting the original works related to titania photocatalysis: A review of papers in the early stage of photocatalysis studies. Electrochemistry 2014, 82, 414-425. [CrossRef]

6. Fujishima, A.; Honda, K. Electrochemical photolysis of water at a semiconductor electrode. Nature 1972, 238, 37-38. [CrossRef] [PubMed]

7. Böttger, H.; Bryksin, V.V. Hopping conductivity in ordered and disordered solids (I). Phys. Stat. Solid B 1976, 78, 9-56. [CrossRef]

8. Nitta, A.; Takase, M.; Takashima, M.; Murakami, N.; Ohtani, B. A fingerprint of metal-oxide powders: Energy-resolved distribution of electron traps. Chem. Commun. 2016, 52, 12096-12099. [CrossRef] [PubMed]

9. Torimoto, T.; Nakamura, N.; Ikeda, S.; Ohtani, B. Discrimination of active crystalline phase in anatase-rutile mixed titanium(IV) oxide photocatalysts through action spectrum analyses. Phys. Chem. Chem. Phys. 2002, 4, 5910-5914. [CrossRef]

10. Torimoto, T.; Aburakawa, Y.; Kawahara, Y.; Ikeda, S.; Ohtani, B. Light intensity dependence of the action spectra of photocatalytic reactions with anatase titanium(IV) oxide. Chem. Phys. Lett. 2004, 392, 220-224. [CrossRef]

11. Nishijima, K.; Ohtani, B.; Yan, X.; Kamai, T.-A.; Chiyoya, T.; Tsubota, T.; Murakami, N.; Ohno, T. Incident light dependence for photocatalytic degradation of acetaldehyde and acetic acid on S-doped and N-doped $\mathrm{TiO}_{2}$ photocatalyst. Chem. Phys. 2007, 339, 64-72. [CrossRef]

12. Yan, X.; Ohno, T.; Nishijima, K.; Abe, R.; Ohtani, B. Is methylene blue an appropriate substrate for a photocatalytic activity test? A study with visible-light responsive titania. Chem. Phys. Lett. 2006, 429, 606-610. [CrossRef]

13. Abe, R.; Shinmei, K.; Koumura, N.; Hara, K.; Ohtani, B. Visible-light-induced water splitting based on two-step photoexcitation between dye-sensitized layered niobate and tungsten oxide photocatalysts in the presence of triiodide/iodide shuttle redox mediator. J. Am. Chem. Soc. 2013, 135, 16872-16884. [CrossRef] [PubMed]

14. Bennett, B. Logically Fallacious: The Ultimate Collection of over 300 Logical Fallacies. Available online: https://www.logicallyfallacious.com/tools/lp/Bo/LogicalFallacies (accessed on 26 November 2016).

15. Ohtani, B.; Zhang, S.-W.; Handa, J.-I.; Kajiwara, H.; Nishimoto, S.-I.; Kagiya, T. Photocatalytic activity of titanium(IV) oxide prepared from titanium(IV) tetra-2-propoxide: Reaction in aqueous silver salt solutions. J. Photochem. Photobiol. A 1992, 64, 223-230. [CrossRef]

16. Prieto-Mahaney, O.-O.; Murakami, N.; Abe, R.; Ohtani, B. Correlation between photocatalytic activities and structural and physical properties of titanium(IV) oxide powders. Chem. Lett. 2009, 38, 238-239. [CrossRef]

17. Ohtani, B.; Prieto-Mahaney, O.-O.; Amano, F.; Murakami, N.; Abe, R. What are titania photocatalysts?-An exploratory correlation of photocatalytic activity with structural and physical properties. J. Adv. Oxidat. Technol. 2010, 13, 247-261. [CrossRef]

18. Ohtani, B.; Prieto-Mahaney, O.-O.; Li, D.; Abe, R. What is Degussa (Evonik) P25? Crystal composition analysis, reconstruction from isolated pure particles, and photocatalytic activity test. J. Photochem. Photobiol. A 2010, 216, 179-182. [CrossRef]

19. Wason, P.C.; Shapiro, D. Natural and contrived experience in a reasoning problem. Quart. J. Exper. Psychol. 1971, 23, 63-71. [CrossRef] 